\title{
A Comparative Transcriptome Analysis of Rhizobium etli Bacteroids: Specific Gene Expression During Symbiotic Nongrowth
}

\author{
Maarten Vercruysse, Maarten Fauvart, Serge Beullens, Kristien Braeken, Lore Cloots, Kristof Engelen, \\ Kathleen Marchal, and Jan Michiels
}

Centre of Microbial and Plant Genetics (CMPG), Katholieke Universiteit Leuven, Kasteelpark Arenberg 20, Heverlee 3001, Belgium

Submitted 31 May 2011. Accepted 21 July 2011.

\begin{abstract}
Rhizobium etli occurs either in a nitrogen-fixing symbiosis with its host plant, Phaseolus vulgaris, or free-living in the soil. During both conditions, the bacterium has been suggested to reside primarily in a nongrowing state. Using genome-wide transcriptome profiles, we here examine the molecular basis of the physiological adaptations of rhizobia to nongrowth inside and outside of the host. Compared with exponentially growing cells, we found an extensive overlap of downregulated growth-associated genes during both symbiosis and stationary phase, confirming the essentially nongrowing state of nitrogen-fixing bacteroids in determinate nodules that are not terminally differentiated. In contrast, the overlap of upregulated genes was limited. Generally, actively growing cells have hitherto been used as reference to analyze symbiosis-specific expression. However, this prevents the distinction between differential expression arising specifically from adaptation to a symbiotic lifestyle and features associated with nongrowth in general. Using stationary phase as the reference condition, we report a distinct transcriptome profile for bacteroids, containing 203 induced and 354 repressed genes. Certain previously described symbiosis-specific characteristics, such as the downregulation of amino acid metabolism genes, were no longer observed, indicating that these features are more likely due to the nongrowing state of bacteroids rather than representing bacteroid-specific physiological adaptations.
\end{abstract}

Rhizobia lead a dual life, either as saprophytes in the soil or as nitrogen-fixing symbionts in root nodules on their leguminous host plant (Fauvart and Michiels 2008; Gibson et al. 2008; Michiels et al. 1991). In the rhizosphere, root exudate flavonoids induce the synthesis of Nod factors by most known rhizobia, which, in turn, cause the initiation of root nodule formation on the host plant. However, host-specific Nod factors are not always essential for inducing nodules, as some photo-

Current address for M. Vercruysse: Department of Biology, Massachusetts Institute of Technology (MIT), Cambridge, MA 02139, U.S.A.

Corresponding author: Jan Michiels;

E-mail: Jan.Michiels@biw.kuleuven.be

Nucleotide sequence data were deposited in the National Center for Biotechnology Information GEO database and can be accessed through accession numbers GSE25094, GSM462178, GSM462180, and GSM462187

* The $e$-Xtra logo stands for "electronic extra" and indicates that three supplementary tables and one supplementary figure are published online. synthetic Bradyrhizobium strains were shown to use a Nod factor-independent strategy (Giraud et al. 2007). Bacteria infect the nodules through infection threads and enter the plant cell cytoplasm by a process that resembles endocytosis, in which a host-derived membrane surrounds them. The bacteria in these compartments, called symbiosomes, subsequently differentiate into nitrogen-fixing bacteroids that reduce atmospheric nitrogen into ammonium, used as a source of nitrogen by the host (Gibson et al. 2008). Mature bacteroids no longer divide and are adapted to the microaerobic environment of the nodule and to specific carbon and nitrogen sources. In the soil, near-starvation conditions, competition with other microbial populations, or fluctuating and adverse environmental conditions often result in bacterial nongrowth (Navarro Llorens et al. 2010). Hence, in both free-living and host-associated nitrogen-fixing conditions, rhizobia primarily reside in a nongrowing state. To cope with the long periods of nongrowth either inside or outside of the host, rhizobia have evolved sophisticated regulatory networks and adaptation mechanisms.

Several studies have examined the transcriptome and proteome of Sinorhizobium meliloti in response to various freeliving stress conditions, including nutrient limitation, microaerobic conditions, oxidative stress, and osmotic stress (Becker et al. 2004; Chen et al. 2003; Djordjevic et al. 2003; Krol and Becker 2004; Rüberg et al. 2003). Furthermore, the analysis of gene and protein expression levels during symbiosis of $S$. meliloti with Medicago sativa or Medicago truncatula has provided insight into the development and function of bacteroids from indeterminate nodules (Ampe et al. 2003; Barnett et al. 2004; Becker et al. 2004; Capela et al. 2006; Djordjevic 2004). More recently, gene expression during symbiosis of Rhizobium leguminosarum with Pisum sativum or Vicia cracca, also forming indeterminate nodules, was analyzed with the emphasis on metabolic adaptation of the bacteria (Karunakaran et al. 2009). Lastly, the nitrogen-fixing symbiosis between Lotus japonicus and Mesorhizobium loti or Bradyrhizobium japonicum, characterized by the formation of determinate nodules, was studied using macro- and microarrays, respectively (Koch et al. 2010; Pessi et al. 2007; Uchiumi et al. 2004). Indeterminate nodules maintain a persistent meristem and contain bacteroids in different stages of differentiation. The mature nitrogen-fixing bacteroids are terminally differentiated and eventually undergo senescence in the nodule. In contrast, determinate nodules have no meristem and contain a homogeneous population of bacteroids at a similar level of development (Gage 2004). These mature bacteroids are not terminally differentiated, enabling them to dedifferentiate in the soil when the nod- 
ule decomposes (Oke and Long 1999). All of the studies cited above, except for Capela and associates (2006), solely use gene expression profiles of actively growing bacteria as a reference to determine symbiotic gene expression. Problematically, this inevitably introduces a bias when describing the transcriptome of nongrowing cells. To remedy this, we here explore the use of transcription profiles obtained from stationary-phase cells as an alternative strategy.

In this study, we identify adaptations of Rhizobium etli bacteroids that are condition specific by a transcriptome analysis comparing gene expression in mature nitrogen-fixing bacteroids with that in free-living cells from exponential- as well as stationary-phase cultures. In addition, we determine to what extent gene expression of not terminally differentiated bacteroids reflects a nongrowing state. Our results show that both symbiotic and stationary-phase states share most of the repressed genes characteristic of nongrowth but differ strongly in terms of induced genes. Transition from growth to nongrowth, therefore, requires the repression of a common core of genes presumably involved in active growth, while activation of condition-specific sets of genes results in adaptation to the particular challenges posed by nongrowth in either the free-living or symbiotic state. To better understand the specific physiology of bacteroids of the R. etli-Phaseolus vulgaris symbiosis, we used stationary-phase cells as an alternative reference. Hence, the use of gene expression data of stationary-phase bacteria as a reference condition, in addition to the commonly used exponential-phase data, is a valuable strategy to obtain symbiosis-specific expression profiles.

\section{RESULTS AND DISCUSSION}

In order to study global gene expression of $R$. etli CFN42 during symbiosis with its host plant, $P$. vulgaris, or the common bean plant, in comparison with free-living cells, we used a custom-design genome-wide tiling array (Vercruysse et al. 2010). Total RNA samples for transcriptional profiling were obtained from three-week-old bacteroids and from aerobically grown cultures in minimal medium, with succinate as carbon source sampled during exponential and stationary phase. In the following sections, a comparative analysis of expression pat-

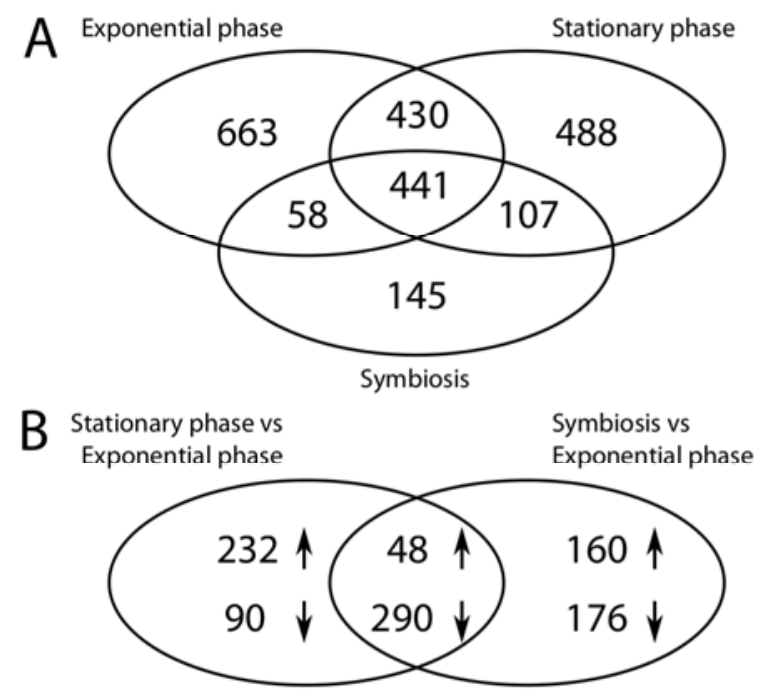

Fig. 1. Gene expression overview. Venn diagrams representing detectable gene expression. A, The number of expressed genes in each condition and the overlap between the different conditions. B, The differentially expressed genes common to both stationary phase and symbiosis or specific to either state, identified by comparing the datasets. Upward- and downward-oriented arrows indicate gene induction and repression, respectively. terns of symbiotic and free-living rhizobia is discussed with special emphasis on which free-living state to use as reference. Details on the differential gene-expression levels, grouped based on predicted functional role and category, are provided in Supplementary Table S1.

\section{Gene expression overview.}

A total of 1,466 and 751 genes are significantly expressed during stationary phase and symbiosis, respectively, compared with 1,592 genes during exponential phase (Fig. 1A). These results show that stationary-phase cells, even though no longer growing at the time of sampling, are not characterized by a significant decrease in the number of expressed genes. However, a distinct difference between both free-living states is observed, as 663 and 488 genes are exclusively expressed during exponential and stationary phase, respectively. In addition, approximately half as many genes are expressed during symbiosis compared with stationary phase, of which about 14\% (107 of 751) are expressed exclusively in both conditions and about $20 \%$ (145 of 751) are symbiosis specific. The fraction of expressed genes located on the six megaplasmids is clearly higher during stationary phase and symbiosis, i.e., 35 and $37 \%$ respectively, compared with exponential growth (21\%). During symbiosis, half of the expressed plasmid genes are located on the symbiotic plasmid, compared with $27 \%$ during stationary phase. This suggests that a significant number of plasmid genes are required for adaptation to each nongrowing state, whereas the housekeeping genes required for active growth are primarily located on the chromosome.

The microarray data were confirmed by analysing the expression levels of 10 representative genes using reverse transcription quantitative polymerase chain reaction (RT-qPCR). These include four genes that are upregulated during symbiosis and stationary phase ( $h m g A / \mathrm{CH} 01746, \mathrm{CH} 01745$, ibpA, $n d i A)$, three genes upregulated exclusively during symbiosis (aqpZ, casA, aptD), and three genes upregulated specifically during stationary phase (aglE, $\operatorname{cox} P$, osm $C$ ). For each gene, two different ratios are plotted versus the respective microar-

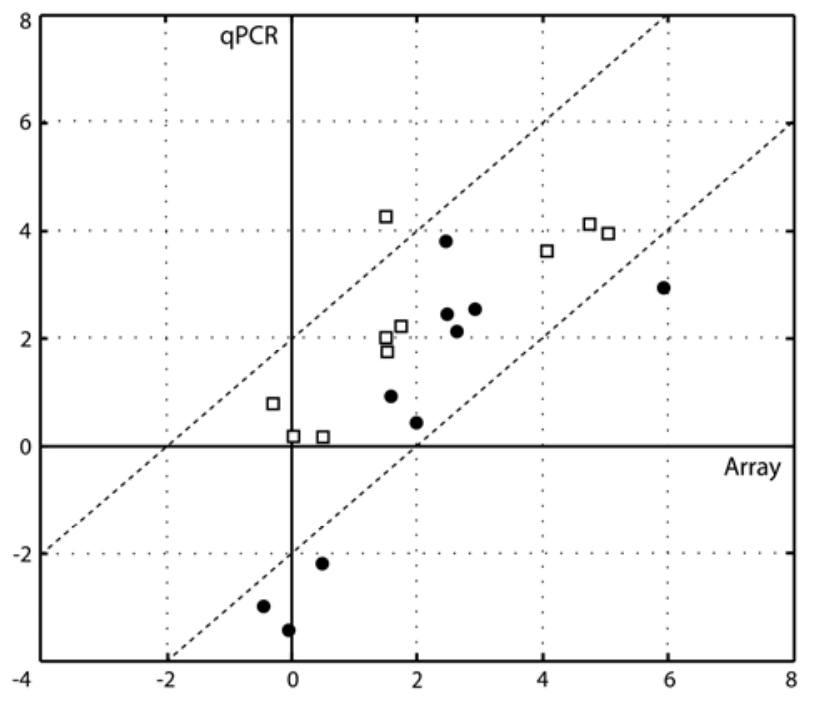

Fig. 2. Reverse transcription quantitative polymerase chain reaction (RTqPCR) validation of the microarray data. Expression of 10 genes was determined using RT-qPCR during the early exponential phase, the stationary phase, and symbiosis three weeks after inoculation. The expression ratios obtained by RT-qPCR ( $y$ axis) and microarrays ( $x$ axis) for each gene were $\log _{2}$-transformed and were plotted against each other for comparison. Filled black dots represent stationary phase vs. early exponential phase and empty black squares represent symbiosis vs. early exponential phase. 
ray ratios, i.e., stationary phase or symbiosis vs. exponential phase (Fig. 2; Supplementary Table S2). This shows that the array data agree well with the RT-qPCR data.

Gene repression during symbiosis and stationary phase, using the exponential phase as reference condition.

Once taken up by host cells, bacteria differentiate into nitrogen-fixing bacteroids and survive in symbiosome compartments. Under free-living conditions, bacteria progress into a stationary phase in response to nutrient limitation or other stressful conditions. In order to better understand to what extent the dramatic shift in gene expression in planta resembles that of free-living nongrowth, we first compared gene expression of mature $R$. etli bacteroids and stationary-phase cells using exponentially growing cells as the reference condition.

A slowdown of cellular activity is characterized by a downregulation of gene expression. Of the 660 genes differentially expressed $\left(-1 \geq \log _{2} \geq 1\right)$ during stationary phase, $58 \%$ are downregulated, whereas $69 \%$ (674 of 751) are downregulated during symbiosis compared with actively growing cells. Moreover, approximately half of both the stationary-phase and symbiotic genes are similarly regulated in both conditions (338 genes), of which $86 \%$ (290 of 338) are downregulated compared with actively growing cells (Fig. 1B). When classified according to functional categories, this extensive overlap of downregulated differentially expressed genes is observed as well (Fig. 3A). Therefore, the general gene repression in mature $R$. etli bacteroids is comparable to that of nongrowing stationary-phase cells. Capela and associates (2006) reported a similar observation for $S$. meliloti bacteroids and assumed the strong reduction of gene expression in mature bacteroids resulted from their growth arrest. $S$. meliloti induces nodules of the indeterminate type, while $R$. etli forms determinate type nodules. Hence, the expression profile of bacteroids resembles that of nongrowing cells, regardless of whether they are terminally differentiated or not.

Most of the common downregulated genes of $R$. etli belong to growth-associated processes. These include 21 genes of the amino-acid metabolism category, including his $B$, lys $C$, leuD, and trpF, nine lipid metabolism genes, including $a c p X L, a c c C$, and $f a b Z$, eight nucleotide metabolism genes, including $n d k$, $n r d I F E$, and purS, 11 motility genes, including flgE, flgG, and $m o t A$, as well as 21 genes involved in cell envelope biosynthesis, including lys $M, l p x D$, and $p s s N$.

Growth arrest is clearly illustrated by the downregulation of genes involved in cell division. parA2 and parB, encoding chromosome segregation proteins, as well as all the plasmid segregation and replication genes $\operatorname{rep} A, \operatorname{rep} B$, and repC are not
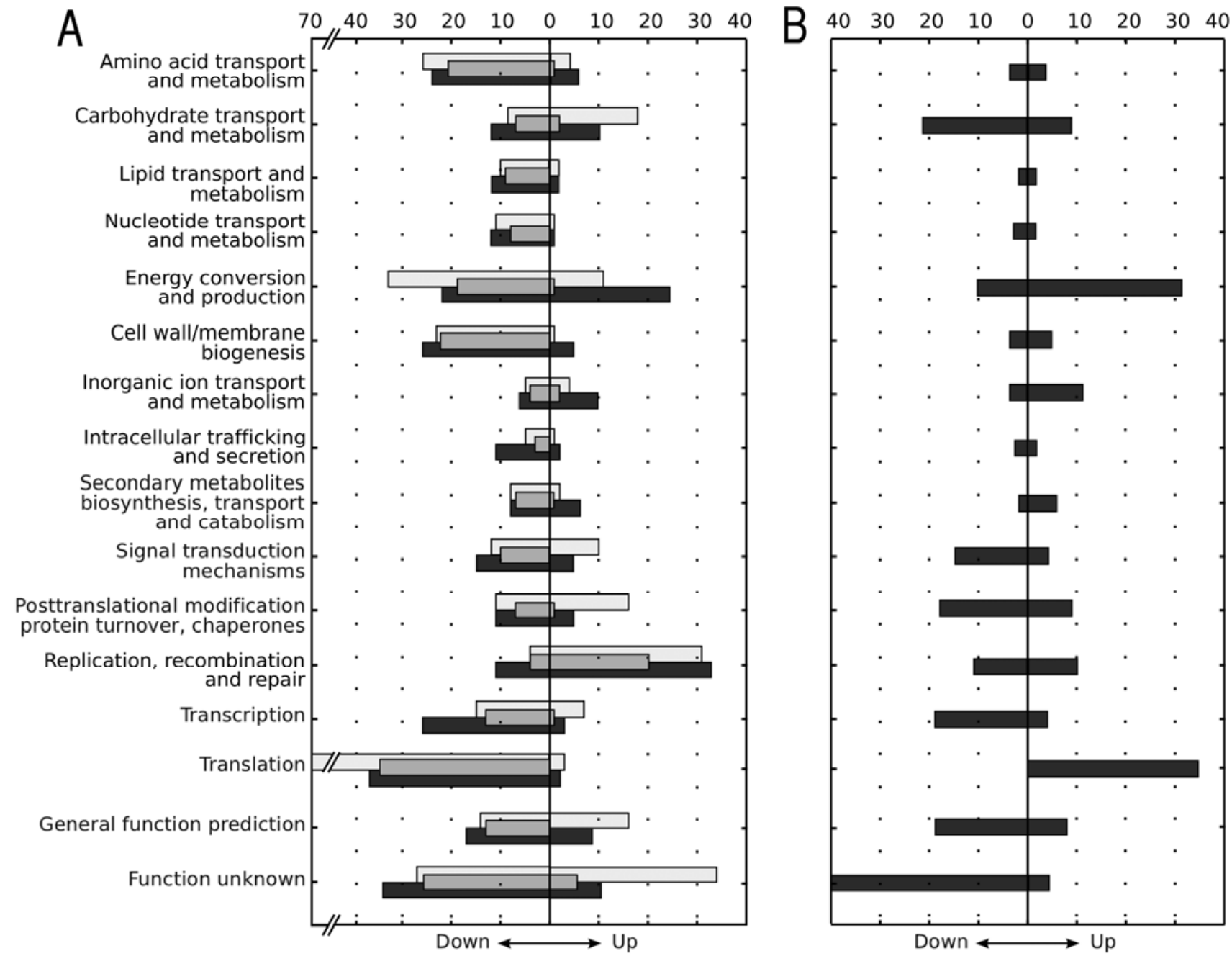

Fig. 3. Differentially expressed genes grouped by functional categories. The number of up- and downregulated genes during during one or both the stationary phase or symbiosis are shown per functional category. The functional categories of the RhizoBase database were used. A, The differential gene expression using the exponential phase as reference condition. Light gray bars represent stationary phase versus exponential phase, black bars represent symbiosis versus exponential phase, and dark gray bars represent the overlapping fraction of genes. B, The differential gene expression in symbiosis using the stationary phase as reference condition. 
expressed during either condition, with the exception of repA $b$ and repAd. RepC was shown to be essential for plasmid replication, while RepA and RepB are involved in plasmid partitioning (Soberón et al. 2004).

Since the level of proteins associated with the translational machinery strongly correlates with growth rate, the observed downregulation of many ribosomal and other translation genes is also in agreement with growth arrest. For example, expression of the genes coding for 23 ribosomal proteins, the translation elongation factor $\mathrm{P}$, the ribosome recycling factor Rrf, and translation initiation factors 1 and 3 is reduced in both nongrowing conditions. However, the translational downregulation partially differs in bacteroids compared with stationary-phase cells as an additional 31 ribosomal proteins and three elongation factors (fusA2, tufA, and tufB) are downregulated over twofold only in stationary phase, while 33 of all 50 tRNAs are downregulated during symbiosis specifically. This is consistent with the previous observation that half of the ribosomal proteins are downregulated in $R$. leguminosarum and B. japonicum bacteroids compared with mid-log phase (Karunakaran et al. 2009; Pessi et al. 2007). In addition, expression of several genes encoding key transcriptional proteins, such as the housekeeping sigma factor SigA, the transcription termination factor Rho, the transcription antitermination factor NusG, and the RNA polymerase omega subunit RpoZ are downregulated during both conditions, reflecting the reduction in global transcriptional activity.

Nongrowth is also characterized by a decreased energy metabolism as is clearly observed during stationary phase by a downregulation of genes implicated in oxidative phosphorylation (nиоA, пиоB, пиоC, пиоD, пиоE, nио, , nиоH, and $s d h B$ ) and genes encoding the $\mathrm{F}_{0} \mathrm{~F}_{1}$ ATP synthase subunits. Even though symbiotic nitrogen fixation is a highly energy-demanding process, the former energy metabolism genes are also downregulated during symbiosis. A similar downregulation was observed in $R$. leguminosarum and $S$. meliloti (Barnett et al. 2004; Karunakaran et al. 2009). The energy demand between stationary-phase cells and bacteroids might differ, as different respiratory systems are active (discussed below). Still, the extent of downregulation of energy metabolism genes is very similar. This further illustrates a similar drop in cellular activity.

In all, the comparison of symbiotic and stationary-phase gene expression, using actively growing cells as a reference, demonstrates an extensive overlap of commonly repressed growth-associated genes.

Gene induction during symbiosis, using the stationary phase as the reference condition.

The transcriptome of actively growing cells, either in complex or minimal medium, is the standard reference condition to analyze differential gene expression during symbiosis and to identify symbiosis-specific genes (Ampe et al. 2003; Barnett et al. 2004; Becker et al. 2004; Karunakaran et al. 2009; Pessi et al. 2007). Compared with the exponential phase, we identified 208 upregulated genes out of the 751 genes expressed during symbiosis. Similar numbers were reported in studies of $S$. meliloti, B. japonicum, and $R$. leguminosarum nitrogen-fixing bacteroids compared with mid-exponential phase cells. Becker

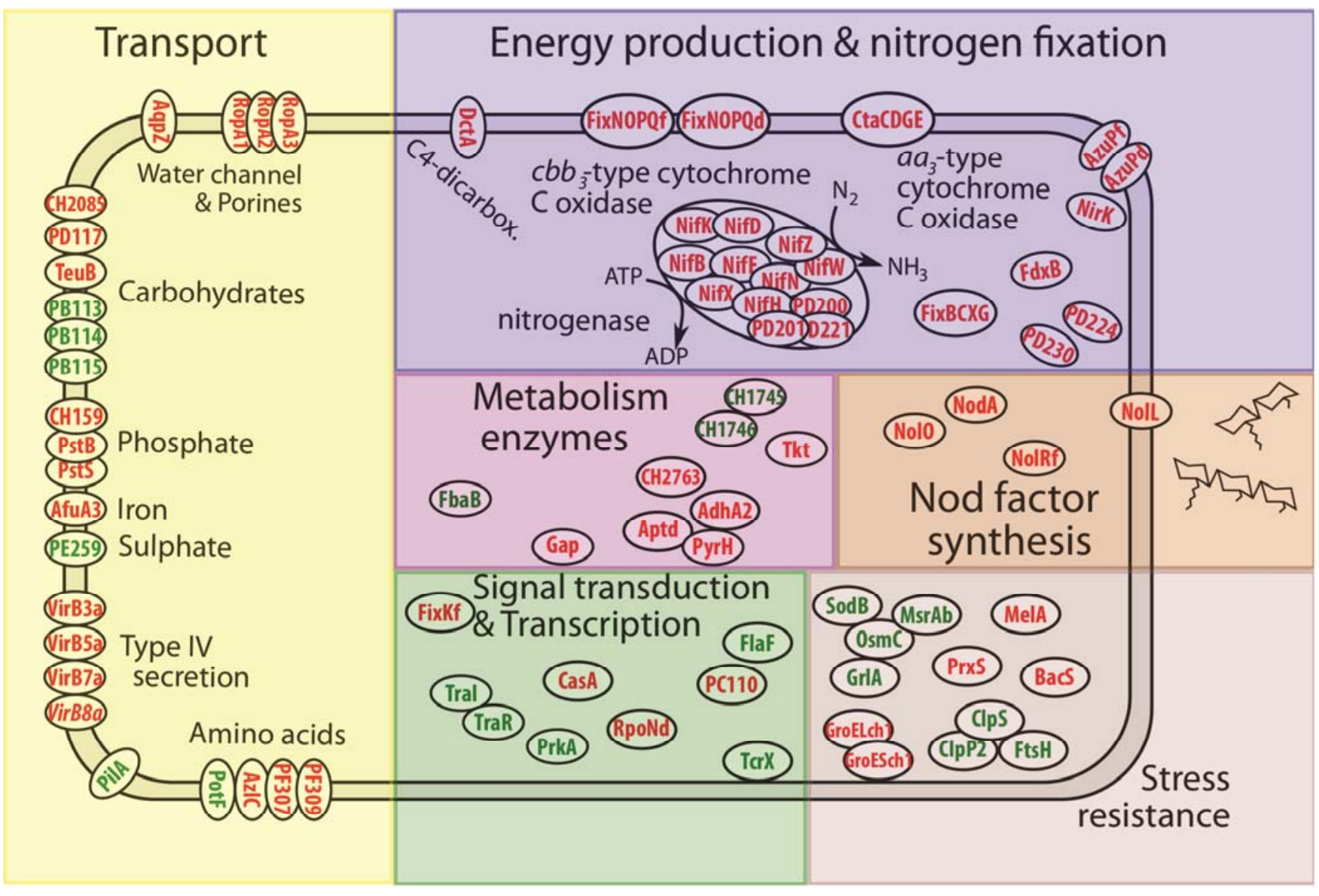

Fig. 4. Overview of symbiosis-specific genes in Rhizobium etli bacteroids. An overview of the genes that are differentially regulated during symbiosis compared with stationary-phase cells illustrating symbiosis-specific gene expression. Upregulated genes are shown in red and downregulated but still expressed genes are shown in green. Gene products are grouped according to function and are represented by rounded rectangles. Membrane-associated proteins are drawn over the inner and outer membrane. 
and coworkers (2004) found 982 differentially expressed genes, of which 342 were upregulated in S. meliloti. Djordjevic (2004) detected 358 proteins in S. meliloti. Pessi and associates (2007) reported 692 genes to be upregulated in 21-day-old bacteroids of $B$. japonicum, while Karunakaran and associates (2009) found 276 upregulated genes in R. leguminosarum. However, these studies do not allow the differentiation between genes differentially expressed in response to symbiotic conditions and genes differentially expressed due to nongrowth in general. Such a distinction can be made using stationaryphase cells as the reference condition.

Using free-living stationary-phase bacteria as a reference allowed us to identify 203 genes that are induced during symbiosis and 354 repressed genes (Fig. 3B), showing a distinctly different expression pattern compared with using exponentially growing cells as the reference condition (Fig. 3A). This pattern is characterized by a strong induction of genes involved in energy production, genes encoding ribosomal proteins, and a moderate induction of genes associated with inorganic ion transport, protein folding, and posttranslational modification (detailed discussion of these genes below). Most carbohydratemetabolism genes as well as many genes involved in signal transduction and transcription are downregulated, though the majority of these genes encode hypothetical proteins of unknown function.

Compared with the stationary phase, the induction of genes involved in energy production is prominent. This is because most of the nitrogen-fixation genes are classified within this category, rather than a higher expression of oxidative phosphorylation and ATP synthesis genes. All differentially expressed genes of the translational category are upregulated, unlike when the exponential phase is used as a reference. These include the essential translation elongation factors $\mathrm{G}$ and $\mathrm{Tu}$, which are downregulated in stationary vs. exponential phase, as well as most ribosomal proteins that are not commonly downregulated compared with growing cells. Hence, even though bacteroids resemble nongrowing cells, as shown above, the use of stationary-phase cells as reference condition provides additional insight into the dissimilarity of translational activity.

The distinct symbiotic downregulation of genes belonging to the amino acid, lipid, and nucleotide metabolism categories, along with cell-wall biogenesis and motility genes is no longer observed when using stationary-phase cells as a reference. This shows that the decrease in expression of these genes, as observed in previous studies (Becker et al. 2004; Capela et al. 2006; Karunakaran et al. 2009; Pessi et al. 2007), is due to the nongrowing state of cells rather than a symbiosis-specific adaptive response. Moreover, bacteroids are auxotrophic for branched-chain amino acids (BCAA), i.e., leucine, isoleucine, and valine, as transcription and activity of BCAA biosynthetic enzymes are highly reduced in symbiotic conditions (Prell et al. 2010). The supply of BCAA by the plant through broadspecificity amino-acid transporters is essential for bacteroid development and persistence (Lodwig et al. 2003; Prell and Poole 2006; Prell et al. 2009). However, a similar downregulation of amino-acid biosynthesis genes is also observed in stationary-phase cells. This distinctive expression pattern is no longer observed when symbiotic gene expression is compared with stationary-phase expression. Therefore, the observed amino-acid auxotrophy seems not solely a symbiosis-specific feature but can be attributed to the nongrowing state of cells.

These data reveal that the comparison of the symbiotic transcriptome to that of stationary-phase cells instead of exponentially growing cells allows differentiation between gene expression specifically associated with symbiosis and expression linked to growth arrest in general.
Symbiosis-specific gene expression.

As little overlap is found between the upregulated genes during symbiosis and stationary phase compared with exponential phase, dedicated sets of genes are apparently used by $R$. etli to adapt to particular nongrowing states (Figs. 1B and $3 \mathrm{~A})$. This observation is consistent with previous studies in $S$. meliloti identifying few bacterial genes or proteins expressed in both stationary-phase cultures and nodules (Capela et al. 2006; Djordjevic 2004; Djordjevic et al. 2003). To study symbiosis-specific gene expression in $R$. etli bacteroids, we compared the bacteroid and stationary-phase transcriptome. An overview of the symbiotic genes discussed below is shown in Figure 4.

Characteristic for the mature bacteroid transcriptome profile (Fig. 3B) is the strong induction of the energy conversion category, as it contains most of the genes involved in the nitrogen fixation machinery (nifKD, nifH, and nifBENX encoding, respectively, dinitrogenase, nitrogenase reductase, and iron-molybdenum cofactor synthesis proteins). These genes are, as expected, among the most highly expressed genes (Jones et al. 2007). Also belonging to the energy production category is the highly upregulated C4-dicarboxylate transporter component DctA. This is in line with the observation that the carbon metabolism of bacteroids is essentially that of dicarboxylategrown cells (Karunakaran et al. 2009; Prell and Poole 2006; Yurgel and Kahn 2004). As the bacteroid adapts to a nongrowing state, it changes its respiration state in order to function in the microaerobic nodule. This entails the use of a $c b b_{3}$-type terminal cytochrome with a high oxygen affinity encoded by fixN, fixO, fix $Q$, and fixP. Both copies, located on the symbiotic and $\mathrm{F}$ plasmid, are induced. Interestingly, the $a a_{3}$-type cytochrome c oxidase used by exponentially growing cells, encoded by ctaC, ctaD, ctaG, and ctaE, is also expressed in bacteroids and upregulated compared with stationary-phase cells, as the latter exclusively expresses the alternative terminal oxidase encoded by $\operatorname{cox} P, \operatorname{cox} Q, \operatorname{cox} N$, and $\operatorname{cox} M$ (González et al. 2006; Vercruysse et al. 2011). Other genes encoding electron transfer proteins are upregulated as well, i.e., ferredoxins ( $f d x B$, PD00230, fixX, fixGd), flavoproteins (PD00224, fixB, $f i x C$ ), and pseudoazurines ( $a z u P d, a z u P f)$ (Lopez et al. 2001; Moris et al. 2004). AzuP is involved in the NO detoxification as a possible electron donor for nitrite reductase. The $\mathrm{Cu}-$ containing nitrite reductase NirK was recently shown to be controlled via FixKf, and both were specifically upregulated during symbiosis (Gómez-Hernández et al. 2011). Under the microaerobic conditions of the nodule, the enhancer NifA regulates the nitrogen-fixation genes together with the symbiotic alternative sigma factor $\sigma^{54}$ or RpoN2. Recently, the NifA-RpoN2 regulon of 11-day-old $R$. etli bacteroids was reported, containing 78 genes (Salazar et al. 2010). We found 56 genes of this regulon to be upregulated in 21-day-old bacteroids (Supplementary Table S3), including genes other than nif and fix such as iscN, aptd, bacS, and melA (discussed below). Moreover, 10 genes involved in the synthesis of Nod factors (nodA, nodJ, nodU, nodZ, nolK, nolL, nolO, nolRch, nolRf, and PD00274) are still expressed in mature bacteroids and also in free-living cells. Four of these genes (nodA, nolO, nolRf, and nolL) are induced above the threshold. This is unexpected, as the primary role of Nod factors is during the early stages of infection (Geurts et al. 2005). In the free-living state, genes involved in Nod factor synthesis were previously shown to be induced by flavonoids after $1 \mathrm{~h}$ and no longer detected $24 \mathrm{~h}$ later (Perret et al. 1999). Previous genome-wide studies of $S$. meliloti and $R$. leguminosarum bacteroids observed an elevated expression of nod genes, including nodA, 5 to 8 days after inoculation (Ampe et al. 2003; Capela et al. 2006; Karunakaran et al. 2009). The level of expression decreased in the later stages of 
symbiosis, suggesting that the synthesis of Nod factors is switched off during bacteroid differentiation in indeterminate nodules (Schlaman et al. 1991). Interestingly, Perret and associates (1999) found several genes of Rhizobium sp. strain NGR234 that are differentially expressed between bacteroids in determinate and indeterminate nodules. These include a number of nod genes that were still expressed in determinate nodules, such as noeE and nolL. Hence, the expressed nodulation genes in $R$. etli might play a role during the later stages of bacteroid development and maintenance.

Consistent with the nongrowing state of mature bacteroids, very few genes of the central metabolism, i.e., glycolysis, gluconeogenesis, and Entner-Doudoroff pathway, are detectably expressed in $R$. etli bacteroids. With dicarboxylic acids as carbon source, the tricarboxylic acid (TCA) cycle is expected to be active. Upregulation of all TCA-cycle enzymes was recently shown in 28-day-old bacteroids of $R$. leguminosarum (Karunakaran et al. 2009). However, we found no gene encoding a TCA enzyme to be expressed in $R$. etli bacteroids, except for $s d h C$, encoding the succinate dehydrogenase subunit $\mathrm{C}$ that is expressed at the same level as in exponentially growing cells. In B. japonicum, it was shown that a fully operational TCA cycle is not essential for nitrogen fixation, indicating that part of the cycle is dispensable (Green et al. 2000; ThönyMeyer and Künzler 1996) In $R$. etli, our transcription data suggest the TCA cycle is largely inactive.

Similarly, as a result of a general slowdown in metabolism, few genes belonging to the intermediary metabolism category, i.e., metabolism of carbohydrates, amino acids, lipids, and nucleotides, are differentially expressed in bacteroids as compared with during stationary phase. The two amino-acid transport and metabolism genes most highly expressed in bacteroids are pot $F$ and $\mathrm{CH} 01745$, encoding a periplasmic putrescine transporter subunit and a 4-hydroxyphenylpyruvate dioxygenase, respectively. The latter is involved in the catabolism of phenylalanine and tyrosine. Tyrosine might be a nutrient for $R$. etli bacteroids, as tyrosine was shown to serve as carbon and nitrogen source for S. meliloti (Milcamps and de Bruijn 1999). Additionally, the $\mathrm{CH} 01745$ homolog in B. japonicum, i.e., $\mathrm{B} 110339$, is also a highly abundant bacteroid protein (Koch et al. 2010). Using an integrated transcriptomics-proteomics approach, Koch and coworkers (2010) reported bll0339 to be a host-specific adaptation gene for symbiosis with cowpea. Symbiosis-specific nucleotide metabolism genes are $\mathrm{pyrH}$ and aptd, encoding, respectively, an uridylate kinase and an adenine phosphoribosyltransferase. The latter is located on the symbiotic plasmid and part of the NifA-RpoN2 regulon. The strong repression of lipid metabolism genes and genes involved in the synthesis of surface polysaccharides, including lipopolysaccharides, exopolysaccharides, and capsular polysaccharides, indicates that the bacteroid cell surface is fully differentiated and requires little further maintenance.

Because symbiosis entails an exchange of compounds, transporters play an important role. In B. japonicum, the transport category was overrepresented in the total number of differentially expressed genes (Pessi et al. 2007), and a particular set of ABC proteins was found in S. meliloti (Djordjevic 2004). Different types of transporters were also found expressed in L. japonicus, M. truncatula, and Glycine max nodule cells (Asamizu et al. 2005; Brechenmacher et al. 2008; El Yahyaoui et al. 2004). In total, components of three dicarboxylic acid transporters (dctA, CH03619, PF00068) and 40 ABC transporters were expressed in $R$. etli bacteroids, including the broad-specificity amino acid transporters aapJAMP, braC1, and $b r a D$. Although the latter transporters are important in bacteroids, as they become auxotrophic for BCAA (Prell et al. 2009), they are similarly expressed in stationary-phase cells.
The only two amino acid transporters induced during symbiosis were a BCAA transporter $(a z l C)$ involved in azaleucine resistance and a probable amino acid ABC transporter (PF00307 and PF00309). In case of sugar transport, genes encoding components of 10 CUT1- and CUT2-family saccharide transporters are expressed, of which three genes $(\mathrm{CH} 02085$, teuB, and PD00117) are upregulated. These data suggest that sugar transport might play a role during symbiosis in $R$. etli. Interestingly, the aquaporin aqpZ of $R$. etli is highly upregulated and was recently shown in the cyanobacterium Synechocystis PCC6803 to play an essential role in glucose metabolism (Akai et al. 2011). AqpZ of $R$. etli is $55 \%$ similar to that of Synechocystis PCC6803. By contrast, repression of sugar utilization was reported in $S$. meliloti and $R$. leguminosarum bacteroids (Djordjevic 2004; Karunakaran et al. 2009). Other upregulated membrane components include outer membrane porines (ropAch1, ropAch2, ropBch3) and type IV secretion proteins (virB3a, virB5a, virB7a, virB8a). The pilin and fimbriae subunit PilA was as highly expressed during symbiosis, comparable to most nif genes, though still downregulated compared with stationary phase, as pilA is the most highly expressed proteinencoding gene in $R$. etli during stationary phase (Vercruysse et al. 2011). Consistent with the nitrogen-fixation deficiency of phosphate transporter mutants in S. meliloti (Bardin et al. 1996), three genes encoding phosphate transport components $(\mathrm{CH} 00159$, pstB, pstS) are upregulated as well as afuA3, which is involved in iron uptake (Chin et al. 1996). These inorganic ion transporters are not expressed in free-living bacteria.

Bacteroids operate under stress as they are confronted with the plant immune system, reactive oxygen or nitrogen species (ROS), ethylene, and phenolic compounds (Abramovitch et al. 2006; Jones et al. 2007). The symbiotic sigma factor RpoN2 regulates, in conjunction with NifA, expression of several genes, including genes that are likely involved in the stress adaptation to the symbiotic niche, i.e., melA, $\operatorname{prxS}$, and 15 genes encoding hypothetical conserved proteins. The tyrosinase MelA catalyzes the production of melanin through oxidation of tyrosine. The pigment melanin has been reported to protect bacteria against several stresses, including high temperature, metal stress, and oxidative stress (Nosanchuk and Casadevall 2003). Melanization was shown to play a role in microbial pathogenesis by reducing susceptibility to host defense mechanisms, though nitrogen fixation of a $R$. leguminosarum melA mutant is not affected (Hawkins et al. 1991). The strong expression of $\mathrm{CH} 01745$ (as described above) is consistent with the catabolism of tyrosine as it produces homogentisate from tyrosine, which is the primary precursor of melanin synthesis (Milcamps and de Bruijn 1999). Hence, our data suggest that melanin may play a role in the stress adaptation of mature $R$. etli bacteroids. Furthermore, the high symbiosisspecific induction of prxS, encoding a peroxidase, is in agreement with previous observations (Dombrecht et al. 2005). In contrast to the corresponding single mutants, nitrogen fixation of a prxS katG mutant is strongly impaired (Dombrecht et al. 2005), indicating that PrxS is likely functionally redundant to KatG and needed for a successful symbiosis. Several other antioxidant enzymes, including $\operatorname{sod} B, \operatorname{osm} C$, grlA, and $m r s A b$, are expressed during symbiosis as well, though downregulated compared with stationary phase. Moreover, $\operatorname{prk} A$, encoding a serine kinase, is expressed in bacteroids as well, though at a lower rate than stationary-phase cells. PrkA likely plays a role in the (p)ppGpp-dependent adaptation of the cellular metabolism (Vercruysse et al. 2011).

In response to nutrient limitation, stress, or decreased protein synthesis, cellular proteins can be recycled through proteolysis. However, few degradation enzymes are expressed in mature bacteroids. The proteases $\mathrm{ClpS}, \mathrm{ClpP} 2$, and $\mathrm{FtsH}$, in- 
volved in ATP-dependent proteolysis, are expressed but are downregulated compared with nongrowing free-living cells. The relatively low number of upregulated and expressed genes belonging to the protein turnover category may indicate that little recycling is required in mature bacteroids as compared with stationary-phase cells. Additionally, the refolding of misfolded proteins caused by stress is often assisted by chaperones. No chaperones were specifically expressed during symbiosis, except for the GroEL/S chaperone system. Of the four groELS operons, only groELSch1 was upregulated while groELSch2 and groELSf were expressed at the same level as stationary-phase cells.

\section{Conclusion.}

In the past decade, several genome-wide transcriptome and proteome studies have described differential gene and protein expression during the rhizobia-legume symbiosis or conditions mimicking symbiosis, such as microaerobic conditions. These studies have allowed the identification of many genes involved in the nitrogen-fixing symbiosis. The standard reference condition in these analyses has been cells in the exponential phase, based on the assumption that growing bacteria characterize the free-living bacterial state. Here, we demonstrate that the stationary phase is a valuable reference condition that needs to be taken into account, as it allows the identification of gene expression resulting from a nongrowing state. The comparison of the symbiotic and stationary transcriptome using exponentially growing cells as a reference shows that mature bacteroids are in a general nongrowing state, due to the extensive overlap of downregulated genes associated with growth processes. While not unique to the bacteroid state, this profound downregulation of global gene expression is an essential feature of the physiology of mature bacteroids. Moreover, bacteroids and stationaryphase cells express different sets of genes, probably reflecting the need to cope with disparate requirements, respectively, inside and outside of the host plant. The identification of differentially expressed genes specific to the symbiotic state and not to nongrowth in general is essential in order to help better understand the intricate details of adaptation by bacteria necessary to establish a successful nitrogen-fixing symbiosis. Hence, this detailed analysis of the specific gene expression during symbiotic nongrowth is a valuable data resource for further functional analysis and emphasizes the use of nongrowing cells as a future reference condition when studying the later events of the rhizobia-legume symbiosis.

\section{MATERIALS AND METHODS}

\section{Bacterial strains and growth conditions.}

R. etli CFN42 was grown at $30^{\circ} \mathrm{C}$ in defined acid minimal salts medium (Poole et al. 1994) supplemented with $10 \mathrm{mM}$ $\mathrm{NH}_{4} \mathrm{Cl}$ as nitrogen source and $10 \mathrm{mM}$ succinate as carbon source, while monitoring the optical density (OD) of the culture. Free-living samples were taken at $\mathrm{OD}_{600}=0.3$ and $6 \mathrm{~h}$ after reaching the maximum OD, representing early exponential and stationary phases, respectively (Supplementary Fig. S1). Bacteroid samples were obtained from nodules three weeks after inoculation. Common bean plants ( $P$. vulgaris cv. Limburgse vroege) were cultivated and inoculated as described previously (D’hooghe et al. 1995).

RNA isolation and cDNA synthesis for microarray detection. RNA was isolated as described previously (Vercruysse et al. 2010). In short, the bacterial RNA from cells in exponential or stationary state was stabilized using a phenol-ethanol solution. Pellets were frozen using liquid nitrogen before being stored at $-80^{\circ} \mathrm{C}$. After crushing $10 \mathrm{~g}$ of nodules from 25 plants using liquid nitrogen, mortar, and pestle, bacteroid RNA was stabilized using RNAprotect (Qiagen, Hilden, Germany).

The TRIzol Plus RNA Purification kit (Invitrogen, Carlsbad, CA, U.S.A.) was used for total RNA isolation. PCR (45 cycles) on all samples determined if the subsequent TURBO DNase (Ambion, Austin, TX, U.S.A.) treatment had removed all remaining DNA. In case of the free-living samples, RNA from six different cultures was pooled in order to increase RNA yields and to account for experimental variation. Experion RNA StdSens Chips (Biorad, Hercules, CA, U.S.A.) before and after precipitation allowed us to determine RNA integrity. All samples had an RNA quality indicator value of 10. The NanoDrop ND-1000 was used to asses RNA quantity and purity. The A260/A280 ratio and A260/A230 ratio of all samples were $\geq 2$.

The microarray cDNA was synthesized, using the SuperScript double-stranded cDNA synthesis kit (Invitrogen) and random decamers (Ambion) according to the manufacturer's protocol.

\section{High-density microarray design and data preprocessing.}

A whole-genome tiling array covering the entire $R$. etli genome sequence was used (Gene Expression Omnibus [GEO] GPL9409), and the data were analyzed as described previously (Vercruysse et al. 2010). The tiling array was designed by NimbleGen Systems, Inc. (Madison, WI, U.S.A.), covering the entire genome sequence of $R$. etli CFN42, using about 385,000 60 mer probes with an average spacing of $13 \mathrm{bp}$ from start to start. Samples were hybridized and scanned by NimbleGen. The data were deposited in the National Center for Biotechnology Information GEO database and can be accessed through accession numbers GSE25094, GSM462178, GSM462180, and GSM462187.

First, a robust estimation of the noise in the expression data was carried out to determine the significant levels of gene expression as described previously (Vercruysse et al. 2010). Second, a standard deviation cut-off was determined in order to identify differentially expressed genes (Vercruysse et al. 2010). Third, if the absolute expression ratio of these genes was greater than or equal to $2\left(\log _{2} \geq 1\right)$, the genes were considered induced or repressed. In nature most regulatory responses appear to function using low-level changes, hence this ratio threshold is fitting (Wren and Conway 2006).

\section{RT-qPCR.}

The array data were validated by determining the expression levels of a group of representative genes using SYBR green RT-qPCR, as described previously (Vercruysse et al. 2010). Briefly, single stranded cDNA was synthesized using the SuperScript VILO cDNA synthesis kit (Invitrogen) starting with $2 \mu \mathrm{g}$ of pooled total RNA from six fresh, independent cultures grown under the same growth conditions (early exponential phase and stationary phase) as well as new bacteroid isolates ( 3 weeks after inoculation). A total of $40 \mathrm{ng}$ of cDNA was used in each reaction, with specific primers designed by Primer Express 3.0. All reactions were performed in triplicate.

The reference gene used was 16S RNA (RHE_CH00059), which showed an invariant expression under the experimental conditions. The early exponential phase was used as calibrator condition. Relative gene expression was calculated using the Pfaffl method that corrects for differences in amplification efficiency (Pfaffl 2001).

\section{ACKNOWLEDGMENTS}

This work was supported by grants from the Research Council of the K.U. Leuven (GOA/011/2008) and from the Fund for Scientific Research- 
Flanders (G.0412.10). M. Vercruysse is indebted to the Institute for the Promotion of Innovation through Science and Technology in Flanders (IWT-Vlaanderen).

\section{LITERATURE CITED}

Abramovitch, R. B., Anderson, J. C., and Martin, G. B. 2006. Bacterial elicitation and evasion of plant innate immunity. Nat. Rev. Mol. Cell. Biol. 7:601-611

Akai, M., Onai, K., Kusano, M., Sato, M., Redestig, H., Toyooka, K., Morishita, M., Miyake, H., Hazama, A., Checchetto, V., Szabo, I. Matsuoka, K., Saito, K., Yasui, M., Ishiura, M., and Uozumi, N. 2011. Plasma membrane aquaporin AqpZ is essential for glucose metabolism during photomixotrophic growth of Synechocystis sp. PCC 6803. J. Biol. Chem. 286:25224-25235.

Ampe, F., Kiss, E., Sabourdy, F., and Batut, J. 2003. Transcriptome analysis of Sinorhizobium meliloti during symbiosis. Genome Biol. 4:R15.

Asamizu, E., Nakamura, Y., Sato, S., and Tabata, S. 2005. Comparison of the transcript profiles from the root and the nodulating root of the model legume Lotus japonicus by serial analysis of gene expression. Mol. Plant-Microbe Interact. 18:487-498.

Bardin, S., Dan, S., Osteras, M., and Finan, T. M. 1996. A phosphate transport system is required for symbiotic nitrogen fixation by Rhizobium meliloti. J. Bacteriol. 178:4540-4547.

Barnett, M. J., Toman, C. J., Fisher, R. F., and Long, S. R. 2004. A dualgenome symbiosis chip for coordinate study of signal exchange and development in a prokaryote-host interaction. Proc. Natl. Acad. Sci. U.S.A. 101:16636-16641.

Becker, A., Bergès, H., Krol, E., Bruand, C., Rüberg, S., Capela, D., Lauber, E., Meilhoc, E., Ampe, F., de Bruijn, F. J., Fourment, J., Francez-Charlot, A., Kahn, D., Küster, H., Liebe, C., Pühler, A., Weidner, S., and Batut, J. 2004. Global changes in gene expression in Sinorhizobium meliloti 1021 under microoxic and symbiotic conditions. Mol. Plant-Microbe Interact. 17:292-303.

Brechenmacher, L., Kim, M., and Benitez, M. 2008. Transcription profiling of soybean nodulation by Bradyrhizobium japonicum. Mol. PlantMicrobe Interact. 21:631-645.

Capela, D., Filipe, C., Bobik, C., Batut, J., and Bruand, C. 2006. Sinorhizobium meliloti differentiation during symbiosis with alfalfa: A transcriptomic dissection. Mol. Plant-Microbe Interact. 19:363-372.

Chen, H., Teplitski, M., Robinson, J. B., Rolfe, B. G., and Bauer, W. D. 2003. Proteomic analysis of wild-type Sinorhizobium meliloti responses to $\mathrm{N}$-acyl homoserine lactone quorum-sensing signals and the transition to stationary phase. J. Bacteriol. 185:5029-5036

Chin, N., Frey, J., Chang, C. F., and Chang, Y. F. 1996. Identification of a locus involved in the utilization of iron by Actinobacillus pleuropneumoniae. FEMS (Fed. Eur. Microbiol. Soc.) Microbiol. Lett. 143:1-6.

D'hooghe, I., Michiels, J., Vlassak, K., Verreth, C., Waelkens, F., and Vanderleyden, J. 1995. Structural and functional analysis of the fix LJ genes of Rhizobium leguminosarum biovar phaseoli CNPAF512. Mol. Gen. Genet. 249:117-126.

Djordjevic, M. A. 2004. Sinorhizobium meliloti metabolism in the root nodule: A proteomic perspective. Proteomics 4:1859-1872.

Djordjevic, M. A., Chen, H. C., Natera, S., van Noorden, G., Menzel, C., Taylor, S., Renard, C., Geiger, O., Weiller, G. F., and Sinorhizobium DNA Sequencing Consortium. 2003. A global analysis of protein expression profiles in Sinorhizobium meliloti: Discovery of new genes for nodule occupancy and stress adaptation. Mol. Plant-Microbe Interact. 16:508-524

Dombrecht, B., Heusdens, C., Beullens, S., Verreth, C., Mulkers, E., Proost, P., Vanderleyden, J., and Michiels, J. 2005. Defence of Rhizobium etli bacteroids against oxidative stress involves a complexly regulated atypical 2-Cys peroxiredoxin. Mol. Microbiol. 55:1207-1221.

El Yahyaoui, F., Kuster, H., Ben Amor, B., Hohnjec, N., Puhler, A., Becker, A., Gouzy, J., Vernie, T., Gough, C., Niebel, A., Godiard, L., and Gamas, P. 2004. Expression profiling in Medicago truncatula identifies more than 750 genes differentially expressed during nodulation, including many potential regulators of the symbiotic program. Plant Physiol. 136:3159-3176.

Fauvart, M., and Michiels, J. 2008. Rhizobial secreted proteins as determinants of host specificity in the Rhizobium-legume symbiosis. FEMS (Fed. Eur. Microbiol. Soc.) Microbiol. Lett. 285:1-9.

Gage, D. J. 2004. Infection and invasion of roots by symbiotic, nitrogenfixing rhizobia during nodulation of temperate legumes. Microbiol. Mol. Biol. Rev. 68:280-300.

Geurts, R., Fedorova, E., and Bisseling, T. 2005. Nod factor signaling genes and their function in the early stages of Rhizobium infection. Curr. Opin. Plant Biol. 8:346-352.

Gibson, K. E., Kobayashi, H., and Walker, G. C. 2008. Molecular determi- nants of a symbiotic chronic infection. Annu. Rev. Genet. 42:413-441.

Giraud, E., Moulin, L., Vallenet, D., Barbe, V., Cytryn, E., Avarre, J.-C., Jaubert, M., Simon, D., Cartieaux, F., Prin, Y., Bena, G., Hannibal, L., Fardoux, J., Kojadinovic, M., Vuillet, L., Lajus, A., Cruveiller, S., Rouy, Z., Mangenot, S., Segurens, B., Dossat, C., Franck, W. L., Chang, W.S., Saunders, E., Bruce, D., Richardson, P., Normand, P., Dreyfus, B., Pignol, D., Stacey, G., Emerich, D., Verméglio, A., Médigue, C., and Sadowsky, M. 2007. Legumes symbioses: Absence of Nod genes in photosynthetic bradyrhizobia. Science 316:1307-1312.

González, V., Santamaría, R. I., Bustos, P., Hernández-González, I., Medrano-Soto, A., Moreno-Hagelsieb, G., Janga, S. C., Ramírez, M. A., Jiménez-Jacinto, V., Collado-Vides, J., and Dávila, G. 2006. The partitioned Rhizobium etli genome: Genetic and metabolic redundancy in seven interacting replicons. Proc. Natl. Acad. Sci. U.S.A. 103:38343839.

Gómez-Hernández, N., Reyes-González, A., Sánchez, C., Mora, Y. Delgado, M. J., and Girard, L. 2011. Regulation and symbiotic role of nirK and norC expression in Rhizobium etli. Mol. Plant-Microbe Interact. 24:233-245.

Green, L. S., Li, Y., Emerich, D. W., Bergersen, F. J., and Day, D. A. 2000 Catabolism of alpha-ketoglutarate by a sucA mutant of Bradyrhizobium japonicum: Evidence for an alternative tricarboxylic acid cycle. J. Bacteriol. 182:2838-2844.

Hawkins, F., Kennedy, C., and Johnston, A. 1991. A Rhizobium-leguminosarum gene required for symbiotic nitrogen-fixation, melanin synthesis and normal growth on certain growth media. J. Gen. Microbiol 137:1721-1728

Jones, K. M., Kobayashi, H., Davies, B. W., Taga, M. E., and Walker, G. C. 2007. How rhizobial symbionts invade plants: The SinorhizobiumMedicago model. Nat. Rev. Micro. 5:619-633.

Karunakaran, R., Ramachandran, V. K., Seaman, J. C., East, A. K., Mouhsine, B., Mauchline, T. H., Prell, J., Skeffington, A., and Poole, P. S. 2009. Transcriptomic analysis of Rhizobium leguminosarum biovar viciae in symbiosis with host plants Pisum sativum and Vicia cracca. J. Bacteriol. 191:4002-4014.

Koch, M., Delmotte, N., Rehrauer, H., Vorholt, J. A., Pessi, G., and Hennecke, H. 2010. Rhizobial adaptation to hosts, a new facet in the legume root-nodule symbiosis. Mol. Plant-Microbe Interact. 23:784790.

Krol, E., and Becker, A., 2004. Global transcriptional analysis of the phosphate starvation response in Sinorhizobium meliloti strains 1021 and 2011. Mol. Genet. Genomics 272:1-17.

Lodwig, E. M., Hosie, A. H. F., Bourdès, A., Findlay, K., Allaway, D. Karunakaran, R., Downie, J. A., and Poole, P. S. 2003. Amino-acid cycling drives nitrogen fixation in the legume-Rhizobium symbiosis. Nature 422:722-726.

Lopez, O., Morera, C., Miranda-Rios, J., Girard, L., Romero, D., and Soberón, M. 2001. Regulation of gene expression in response to oxygen in Rhizobium etli: Role of FnrN in fixNOQP expression and in symbiotic nitrogen fixation. J. Bacteriol. 183:6999-7006.

Michiels, J., Vande Broek, A., and Vanderleyden, J. 1991. Molecular cloning and nucleotide sequence of the Rhizobium phaseoli recA gene. Mol. Gen. Genet. 228:486-490.

Milcamps, A., and de Bruijn, F. J. 1999. Identification of a novel nutrientdeprivation-induced Sinorhizobium meliloti gene ( $\mathrm{hmgA}$ ) involved in the degradation of tyrosine. Microbiology 145:935-947.

Moris, M., Dombrecht, B., Xi, C., Vanderleyden, J., and Michiels, J., 2004. Regulatory role of Rhizobium etli CNPAF512 fnrN during symbiosis. Appl. Environ. Microbiol. 70:1287-1296.

Navarro Llorens, J. M., Tormo, A., and Martínez-García, E. 2010. Stationary phase in gram-negative bacteria. FEMS (Fed. Eur. Microbiol. Soc.) Microbiol Rev. 34:476-495.

Nosanchuk, J. D., and Casadevall, A. 2003. The contribution of melanin to microbial pathogenesis. Cell Microbiol. 5:203-223.

Oke, V., and Long, S. R. 1999. Bacteroid formation in the Rhizobium-legume symbiosis. Curr Opin Microbiol 2:641-646.

Perret, X., Freiberg, C., Rosenthal, A., Broughton, W. J., and Fellay, R. 1999. High-resolution transcriptional analysis of the symbiotic plasmid of Rhizobium sp. NGR234. Mol. Microbiol. 32:415-425.

Pessi, G., Ahrens, C. H., Rehrauer, H., Lindemann, A., Hauser, F., Fischer, and H.-M., Hennecke, H. 2007. Genome-wide transcript analysis of Bradyrhizobium japonicum bacteroids in soybean root nodules. Mol. Plant-Microbe Interact. 20:1353-1363.

Pfaffl, M. W. 2001. A new mathematical model for relative quantification in real-time RT-PCR. Nucleic Acids Res. 29:e45.

Poole, P. S., Schofield, N. A., Reid, C. J., Drew, E. M., and Walshaw, D. L. 1994. Identification of chromosomal genes located downstream of $d c t D$ that affect the requirement for calcium and the lipopolysaccharide layer of Rhizobium leguminosarum. Microbiology 140:2797-2809.

Prell, J., and Poole, P. 2006. Metabolic changes of rhizobia in legume nod- 
ules. Trends Microbiol. 14:161-168.

Prell, J., White, J. P., Bourdes, A., Bunnewell, S., Bongaerts, R. J., and Poole, P. S. 2009. Legumes regulate Rhizobium bacteroid development and persistence by the supply of branched-chain amino acids. Proc. Natl. Acad. Sci. U.S.A. 106:12477-12482.

Prell, J., Bourdès, A., Kumar, S., Lodwig, E., Hosie, A., Kinghorn, S., White, J., and Poole, P. 2010. Role of symbiotic auxotrophy in the Rhizobium-legume symbioses. PLoS ONE 5:e13933. Published online

Rüberg, S., Tian, Z.-X., Krol, E., Linke, B., Meyer, F., Wang, Y., Pühler, A., Weidner, S., and Becker, A. 2003. Construction and validation of a Sinorhizobium meliloti whole genome DNA microarray: Genome-wide profiling of osmoadaptive gene expression. J. Biotechnol. 106:255-268.

Salazar, E., Díaz-Mejía, J. J., Moreno-Hagelsieb, G., Martínez-Batallar, G., Mora, Y., Mora, J., and Encarnación, S. 2010. Characterization of the NifA-RpoN regulon in Rhizobium etli in free life and in symbiosis with Phaseolus vulgaris. Appl. Environ. Microbiol. 76:4510-4520.

Schlaman, H. R., Horvath, B., Vijgenboom, E., Okker, R. J., and Lugtenberg, B. J. 1991. Suppression of nodulation gene expression in bacteroids of Rhizobium leguminosarum biovar viciae. J. Bacteriol. 173:4277-4287.

Soberón, N., Venkova-Canova, T., Ramírez-Romero, M. A., Téllez-Sosa J., and Cevallos, M. A. 2004. Incompatibility and the partitioning site of the repABC basic replicon of the symbiotic plasmid from Rhizobium etli. Plasmid 51:203-216.

Thöny-Meyer, L., and Künzler, P. 1996. The Bradyrhizobium japonicum aconitase gene $(a c n A)$ is important for free-living growth but not for an effective root nodule symbiosis. J. Bacteriol. 178:6166-6172.
Uchiumi, T., Ohwada, T., Itakura, M., Mitsui, H., Nukui, N., Dawadi, P. Kaneko, T., Tabata, S., Yokoyama, T., Tejima, K., Saeki, K., Omori, H., Hayashi, M., Maekawa, T., Sriprang, R., Murooka, Y., Tajima, S., Simomura, K., Nomura, M., Suzuki, A., Shimoda, Y., Sioya, K., Abe, M., and Minamisawa, K. 2004. Expression islands clustered on the symbiosis island of the Mesorhizobium loti genome. J. Bacteriol. 186:2439-2448.

Vercruysse, M., Fauvart, M., Cloots, L., Engelen, K., Thijs, I. M., Marchal, K., and Michiels, J. 2010. Genome-wide detection of predicted noncoding RNAs in Rhizobium etli expressed during free-living and hostassociated growth using a high-resolution tiling array. BMC Genomics $11: 53$.

Vercruysse, M., Fauvart, M., Jans, A., Beullens, S., Braeken, K., Cloots, L., Engelen, K., Marchal, K., and Michiels, J. 2011. Stress response regulators identified through genome-wide transcriptome analysis of the (p)ppGpp-dependent response in Rhizobium etli. Genome Biol. 12:R17.

Wren, J. D., and Conway, T. 2006. Meta-analysis of published transcriptional and translational fold changes reveals a preference for low-fold inductions. OMICS 10:15-27.

Yurgel, S. N., and Kahn, M. L. 2004. Dicarboxylate transport by rhizobia. FEMS (Fed. Eur. Microbiol. Soc.) Microbiol Rev. 28:489-501.

AUTHOR-RECOMMENDED INTERNET RESOURCE

RhizoBase database: genome.kazusa.or.jp/rhizobase 